\title{
Dose rate analysis for the diagnostic generic equatorial port plug in the ITER port plug test facility
}

\author{
Haibo Liu ${ }^{1}$, Thierry Cerisier², Ulrich Fischer ${ }^{1}$, Bruno Levesy², Alexander Romannikov ${ }^{3}$, Arkady Serikov ${ }^{1}$ \\ ${ }^{1}$ Karlsruhe Institute of Technology, Hermann-von-Helmholtz-Platz 1, 76344 Karlsruhe, Germany \\ 2ITER Organization, Route de Vinon-sur-Verdon, CS 90 046, 13067 St. Paul Lez Durance Cedex, France \\ ${ }^{3}$ Russian Federation ITER Domestic Agency, Kurchatov sq.1, 123182, Moscow, Russia
}

\section{Introduction}

The port plug test facility (PPTF) in the ITER hot cell building (HCB) will be used to test activated equatorial port plugs. In this work, the R2Smesh approach [1] was used to provide high resolution maps of dose rate distributions in the PPTF and the adjacent rooms.

\section{Neutron flux and activation calculations for the GEPP}

The diagnostic GEPP MCNP model was integrated into the ITER B-lite MCNP model for neutron transport calculation. The GEPP decay gamma source distribution was calculated by R2Smesh with ITER SA2 operation scenario [2] and 30 days cooling.

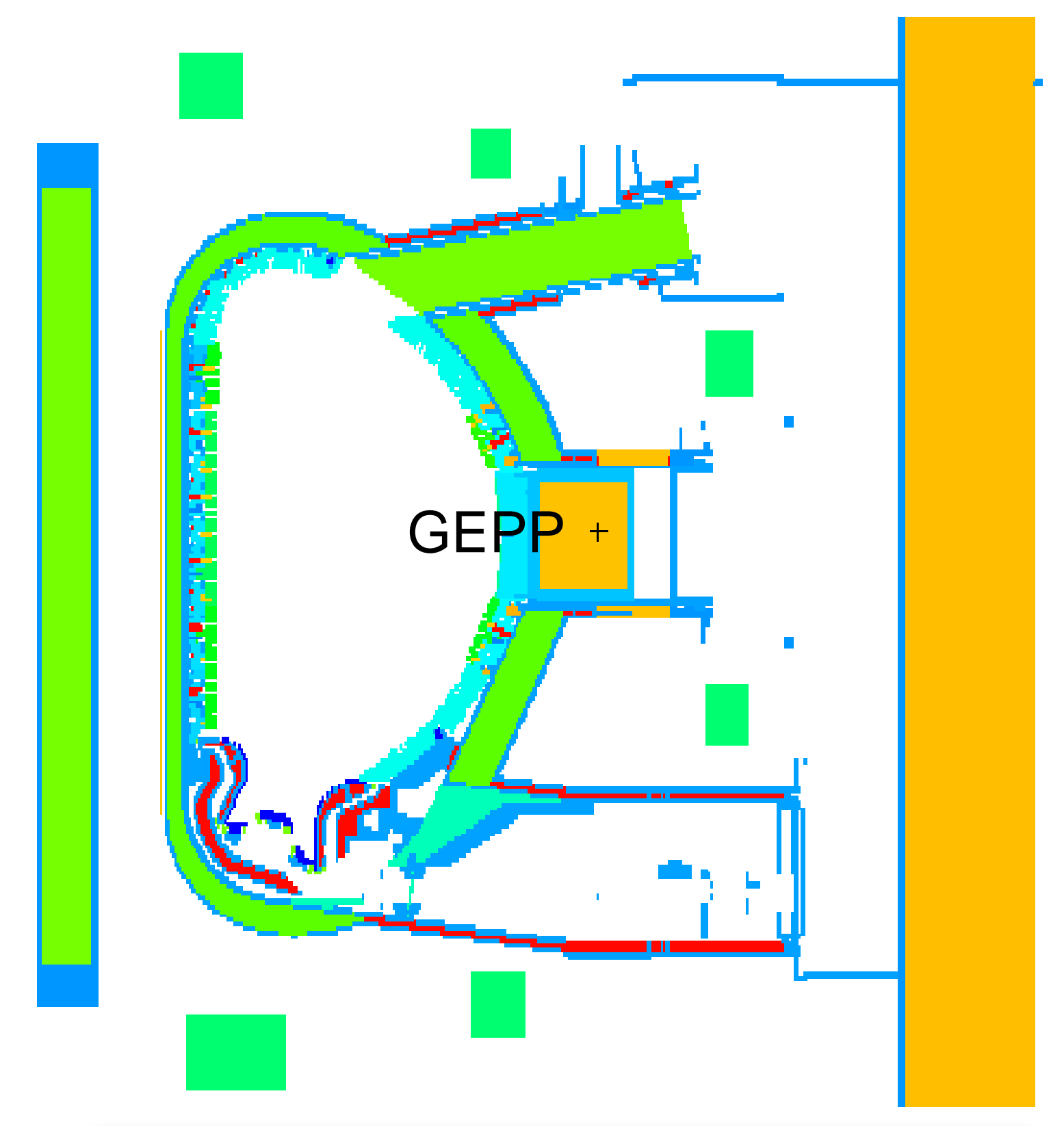

Fig. 1. GEPP in ITER B-lite model

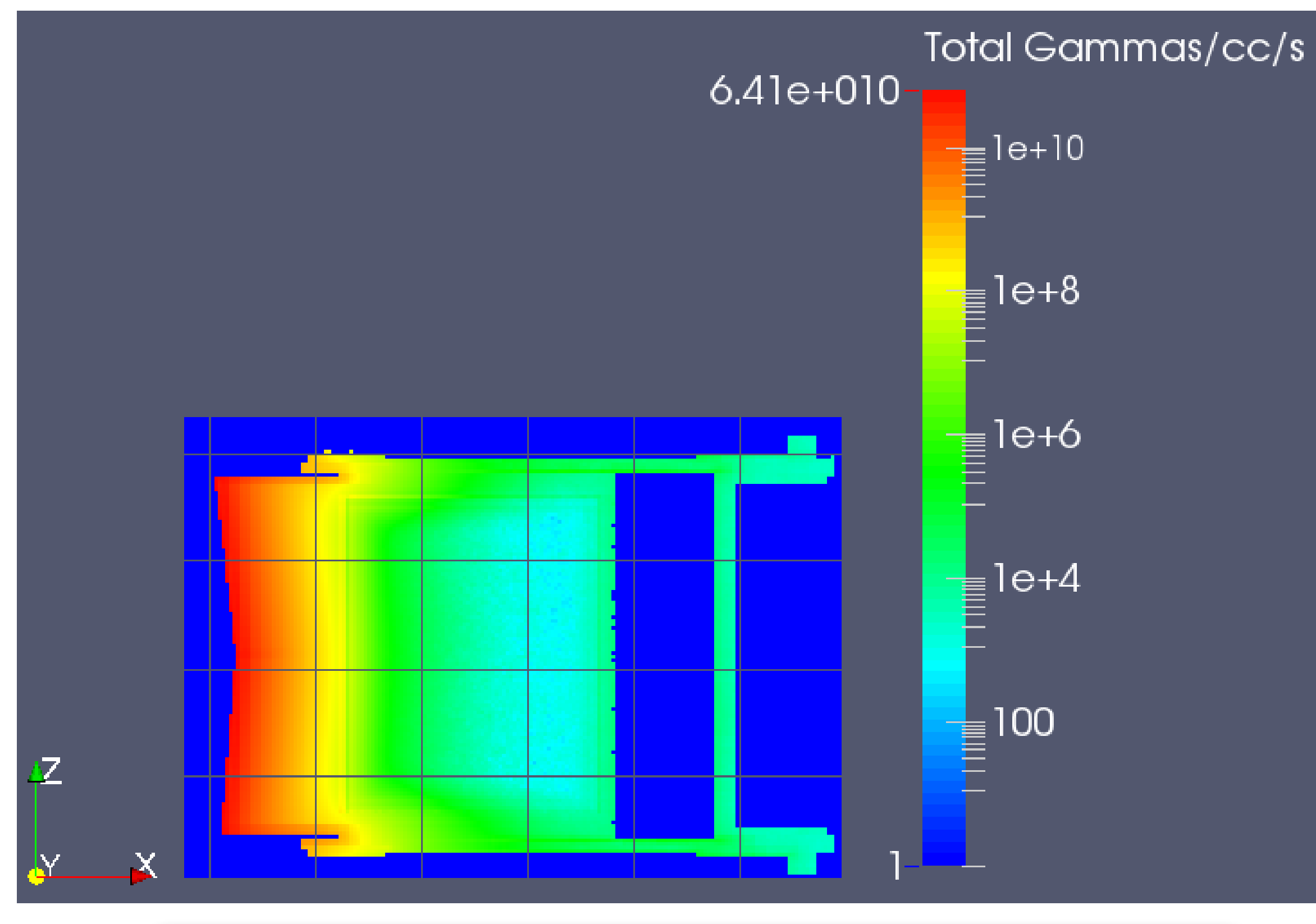

Fig. 2. GEPP decay gamma source distribution

\section{PPTF modeling and dose rate calculation}

A preliminary PPTF\&HCB MCNP model was prepared by the Russia DA. The test tank wall is a $2 \mathrm{~cm}$ thick steel wall. The GEPP module was integrated into this model for dose rate calculation.

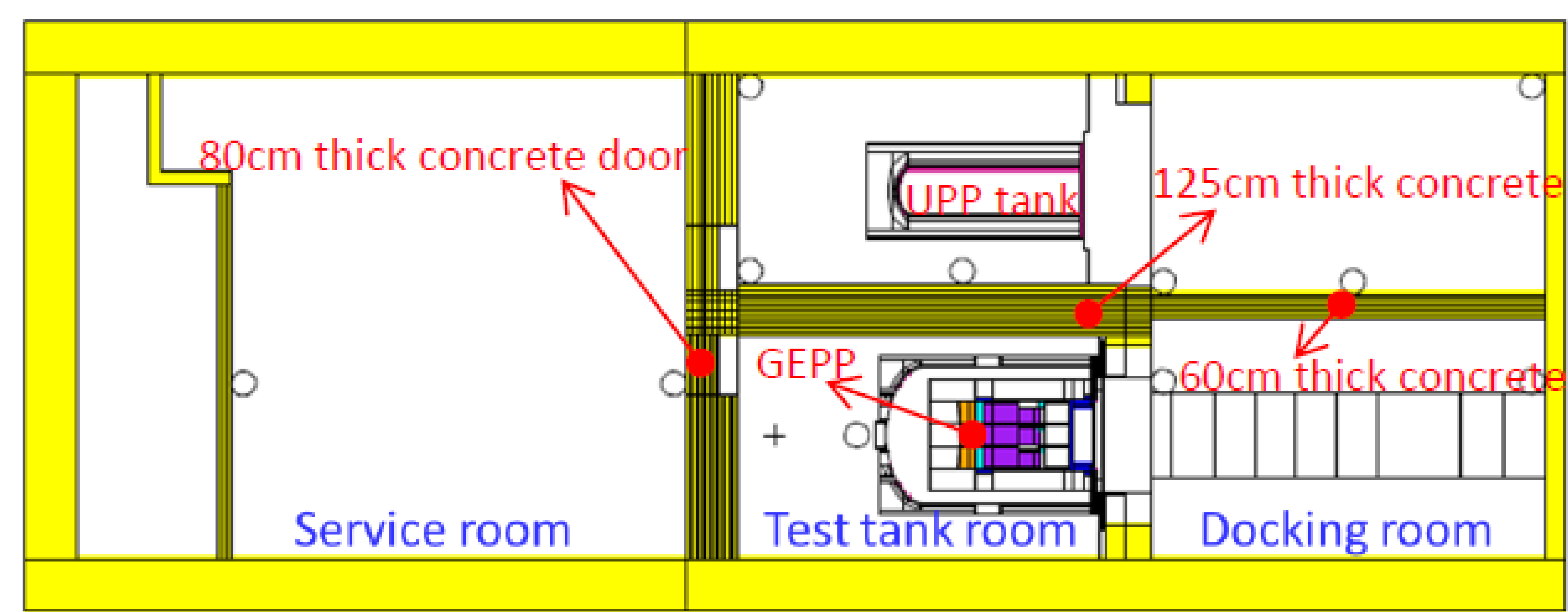

Fig. 3. GEPP integrated with PPTF\&HCB model from the preliminary design

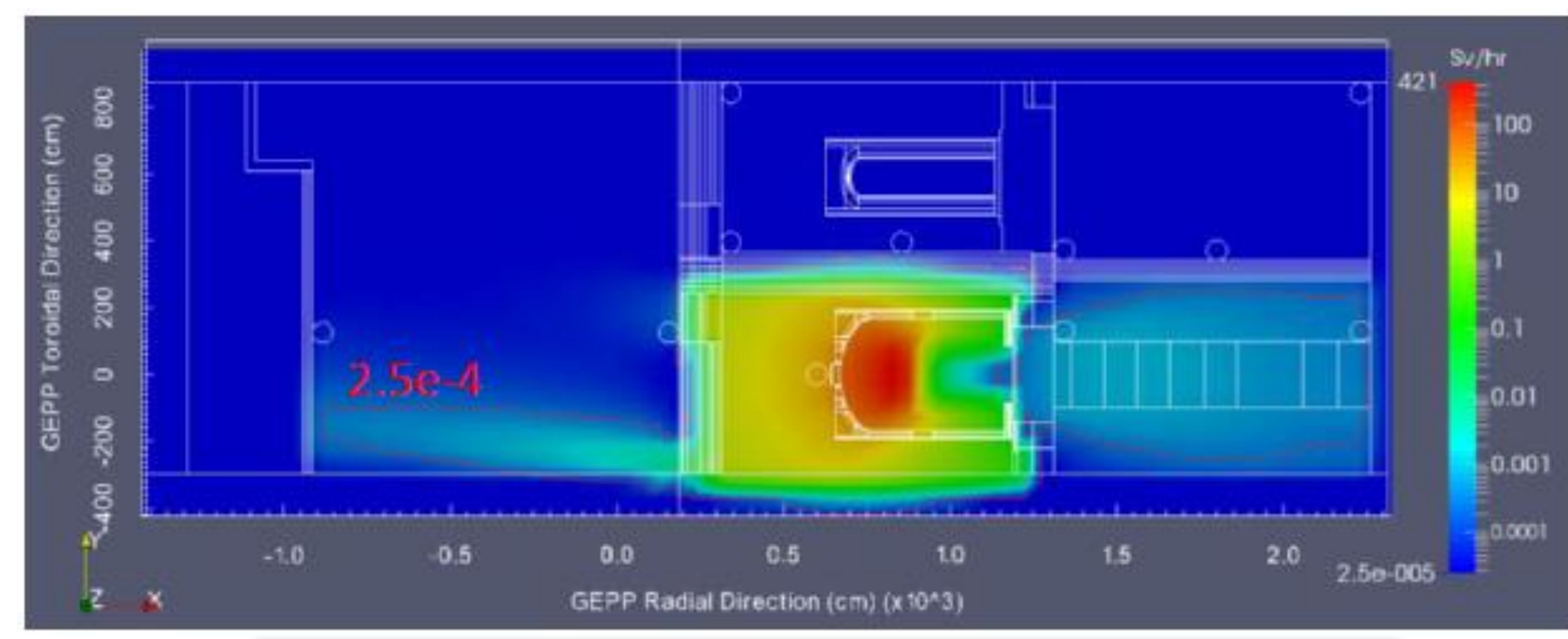

Fig. 4. Dose rate map obtained with the preliminary PPTF design
The dose rate map for the whole building is shown in fig. 4. The dose rates in the service room and docking room are higher than $250 \mu \mathrm{Sv} / \mathrm{hr}$.

\section{Design optimization for improved radiation} shielding

A design optimization was performed to reduce the dose rate. The dose rate map for the optimized design is shown in fig. 6.

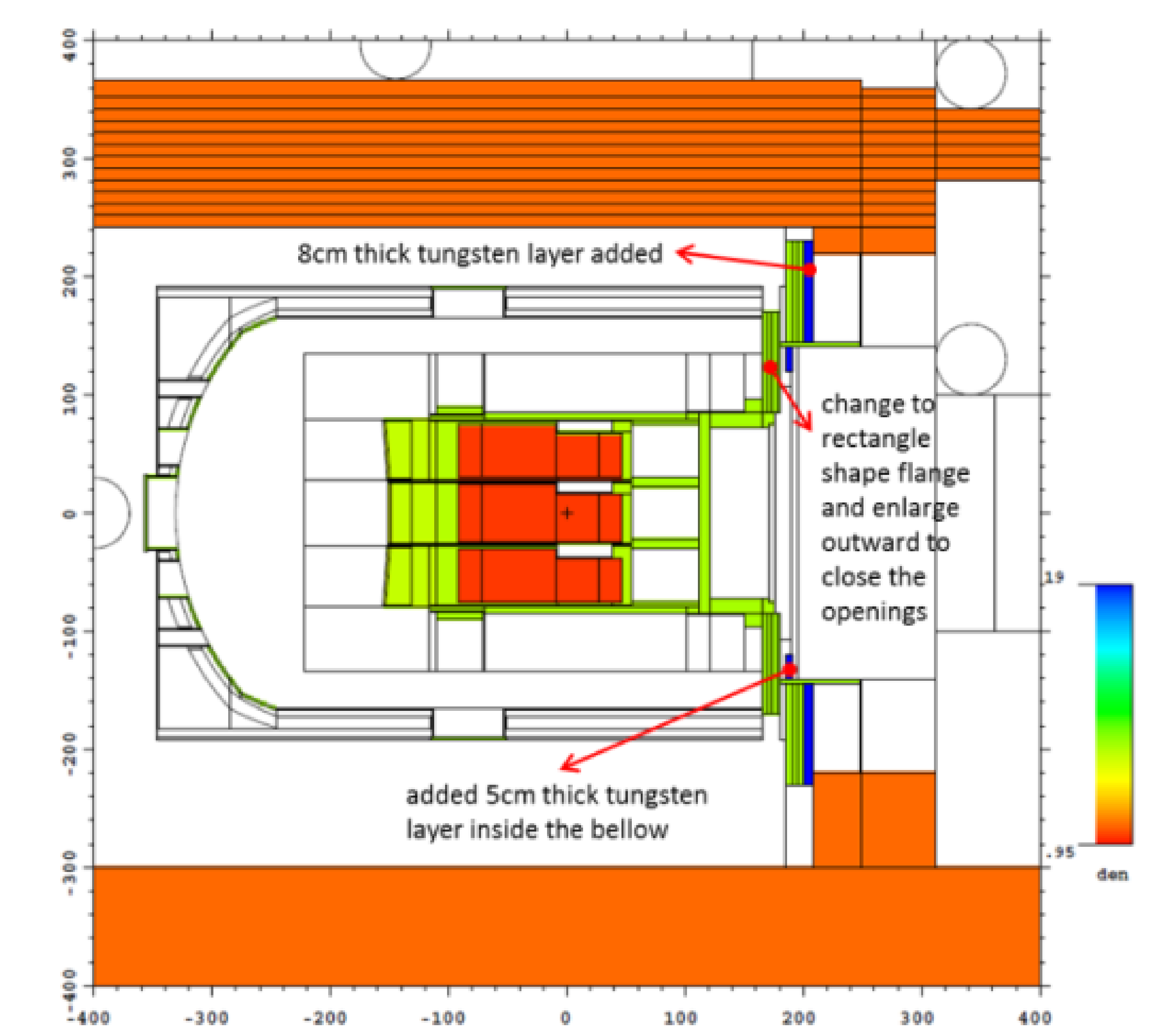

Fig. 5. Test tank area with design modifications applied

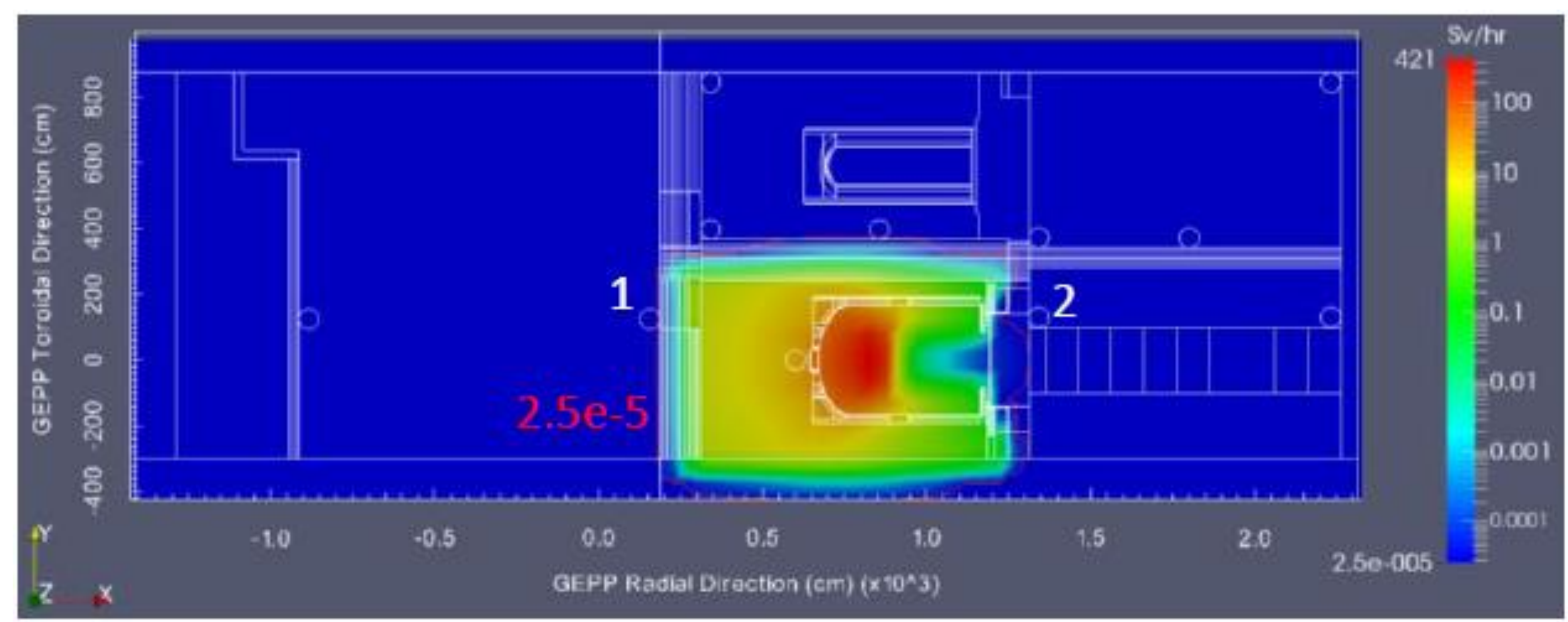

Fig. 6. Dose rate map obtained for the optimized PPTF design

\section{Summary}

This work used the R2Smesh approach to assess the dose rate distribution in the three HCB rooms when the activated diagnostic GEPP is placed in the PPTF. After preliminary analysis and design optimization, the dose rates in the service and the docking rooms are lower than $25 \mu \mathrm{Sv} / \mathrm{hr}$.

\section{Acknowledgments}

The views and opinions expressed herein do not necessarily reflect those of the ITER Organization. 